\title{
Un gène dominant de stérilité mâle chez le hari- cot (Phaseolus vulgaris L.)
}

\author{
Hubert BANNEROT, Joseph-Martin BELL $\left({ }^{*}\right)$, Bernard BOSC $\left({ }^{* *}\right)$ \& Robert CAMUT $\left({ }^{* *}\right)$ \\ I.N.R.A., Station de Génétique et d'Amélioration des Plantes. Centre de Recherches de Versailles, Route de \\ Saint-Cyr, F 78000 Versailles \\ (*) Université de Yaoundé, B.P. 812, Yaoundé, Cameroun \\ (**) Institut de Recherche Vilmorin, La Ménitré, 49250 Beaufort-en-Vallée
}

\begin{abstract}
Plusieurs plantes mâle stériles ont été observées en 1981 et en 1982 dans des générations avancées issues du croisement des variétés Valja $\times$ Starnel. Tous les croisements effectués par la suite entre ces plantes ou leurs descendants mâle stériles et n'importe quel génotype fertile ont donné une descendance $F 1$ en ségrégation pour la stérilité mâle dans des proportions statistiquement compatibles avec $1: 1$.

Les plantes mâle fertiles ont toujours donné des descendances uniformément mâle fertiles.

Ces résultats sont en accord avec l'hypothèse d'un gène dominant commandant l'expression de la stérilité mâle. Les plantes mâle stériles seraient hétérozygotes Ms ms et les plantes mâle fertiles seraient homozygotes ms ms. Des couples isogéniques sont en cours de constitution pour les variétés : Contender, Maxidor, Red Kloud, Copau, EO2, Tz, S102, Emerit, Fortex, Borlotto Gigante. Du fait de l'absence totale de pollen colorable, observée jusqu'à présent chez les mâle stériles (à l'exception des rétrocroisements avec la variété Copau), il semble difficile d'obtenir les homozygotes dominants MsMs et de transférer le gène dans un autre cytoplasme. L'utilisation du caractère pour l'amélioration du haricot est discutée.
\end{abstract}

Nous proposons de dénommer ce gène Ms8.

Mots clés additionnels : sélection récurrente, microgamètogenèse.

Several male-sterile plants were observed in 1981 and 1982 among advanced progeny yielded by the cross of cvs Valja $\times$ Starnel at the Vilmorin Research Institute in La Ménitré. All subsequent crosses between these plants or their male-sterile descendants and any fertile genotype gave rise to an F1 generation that segregated for male sterility in proportions statistically compatible with $1: 1$. Male-fertile plants always uniformly yielded malefertile progeny. These results may be explained by the hypothesis that a dominant gene directs the expression of male sterility. According to this hypothesis, male-sterile plants would be heterozygous Ms ms and male-fertile plants would be homozygous ms ms. Isogenic pairs are currently being constructed for the following cultivars : Contender, Maxidor, Red Kloud, Copau, EO2, Tz, S102, Emerit, Fortex, Borlotto Gigante. Given the complete absence of stainable pollen observed until now in the male-sterile plants (with the exception of the progeny of backcrosses with cv. Copau), it seems difficult to obtain homozygous dominant Ms Ms and to transfer this gene into another cytoplasm. The use of this trait in plant breeding is discussed. We propose to denote the gene Ms8.

Additional key words : recurrent selection, microgametogenesis.

\section{INTRODUCTION}

La découverte de gènes dominants de stérilité mâle a déjà été signalée chez le coton (ALLISON \& FISHER, 1964 ; WEAVER \& ASHLEY, 1971 ; BOWMAN \& WEAVER, 1979 ; TURCOTTE \& FEASTER, 1985), le blé (SASAKUMA et al., 1978) et la laitue (RYDER, 1963). Un cas a également été trouvé récemment chez le choufleur (Y. HERvÉ, communication personnelle). Cette relative rareté constraste avec le nombre très élevé de stérilités mâle géniques récessives déjà décrites chez de nombreuses espèces. Chez Phaseolus vulgaris L., au moins 7 systèmes géniques, tous récessifs, ont déjà été signalés (IBRAHIM et al., 1974 ; AGBO \& WOOD, 1977 ; VAN RHEENEN et al., 1979 ; MUTSCHLER \& BLISS, 1980 ; SINGH et al., 1980 : BASSETT \& HUNG, 1982 ; WYATT, 1984). Nous en avons nous-mêmes observé plusieurs (BANNEROT, 1980). Ces stérilités sont utilisées le plus souvent pour faciliter les croisements, notamment dans les programmes de sélection récurrente qui impliquent un grand nombre d'opérations de croisements contrôlés. 
Cet article rapporte un cas de stérilité mâle dominante chez $P$. vulgaris, qui est à notre connaissance, le premier de cette nature observé chez cette espèce.

\section{MATÉRIEL ET MÉTHODES}

\section{A. Matériel d'origine et étude génétique}

En 1981, dans un champ de sélection de l'Institut de Recherches Vilmorin (IRV), plusieurs plantes mâle stériles furent observées dans une famille F6 issue du croisement Valja $\times$ Starnel, mais aucune descendance ne put être récoltée.

Valja et Starnel sont 2 lignées pures de haricot mangetout de conserve à grain blanc, inscrites au catalogue officiel français, la première a été obtenue par VEB Saat und Pflanzgut (République Démocratique allemande), la seconde est une obtention de l'Institut de Recherches Vilmorin (France).

En 1982, une plante mâle stérile fut également observée dans des familles issues du même croisement semées en serre. Elle fut croisée par une plante fertile sœur. Les 7 plantes F1 issues de ce croisement ( $\left.\mathrm{n}^{\circ} 1\right)$ se répartirent en 5 fertiles et 2 stériles. Les 2 plantes stériles furent croisées par 3 lignées pratiquement fixées (F8 et plus) : Flaveol, 422, 423, donnant respectivement les descendances F1 : A, B, C, qui furent étudiées à l'I.N.R.A. de Versailles (tabl. 1).

TABLEAU 1

Ségrégation en Fl des croisements de départ. Segregation in Fl of first crosses.

\begin{tabular}{lccc}
\hline \multicolumn{1}{c}{ Croisements } & \multicolumn{2}{c}{ Phénotype } & \\
\cline { 2 - 3 } & $\mathrm{mf}$ & $\mathrm{ms}$ & $\mathrm{T}$ \\
\hline $\mathrm{N}^{\circ} 1 \mathrm{croisement} \mathrm{initial}$ & 5 & 2 & 7 \\
$\mathrm{~A}(1.1 \mathrm{~ms} \times$ Flaveol $)$ & 1 & 0 & 1 \\
$\mathrm{~B}(1.2 \mathrm{~ms} \times 422)$ & 2 & 7 & 9 \\
$\mathrm{C}(1.2 \mathrm{~ms} \times 423)$ & 2 & 3 & 5 \\
\hline Totaux & 10 & 12 & 22 \\
\hline \hline
\end{tabular}

$\mathrm{mf}=$ mâle fertile,

$\mathrm{ms}=$ mâle stérile.

En 1983, les 3 plantes mâle stériles issues du croisement $\mathrm{C}$ ont été croisées avec les génotypes figurant au tableau 2 et quelques autres. Pour les génotypes suivants : Contender, Maxidor, Red Kloud, Copau, EO2, TG, S102, Emérite, Fortex, Borlotto Gigante, les rétrocroisements ont été poursuivis en vue de constituer des couples isogéniques ne différant que par le caractère de stérilité mâle.

$\mathrm{Au}$ printemps 1984 , en vue d'une étude génétique plus poussée, un grand nombre de croisements ont été réalisés sur quelques plantes mâle stériles de chaque programme. Ces descendances $\mathrm{F} 1$ ont servi pour l'étude de la ségrégation (tabl. 2). Les descendances F2 issues des plantes F1 mâle fertiles ont été également observées.

\section{B. Culture des plantes}

Les plantes sont cultivées en serre (chauffée et éclairée artificiellement en hiver) dans des pots de 3 ou
TABLEAU 2

Ségrégation en $F 1$ des différents programmes de rétrocroisements. Segregation in $\mathrm{Fl}$ of the different back-cross programmes.

\begin{tabular}{|c|c|c|c|c|c|}
\hline \multirow{2}{*}{ Parents mâles } & \multicolumn{2}{|c|}{ Phénotype } & \multirow[b]{2}{*}{ Total } & \multirow[b]{2}{*}{$x^{2}$} & \multirow[b]{2}{*}{$\mathrm{P}$} \\
\hline & $\mathrm{mf}$ & $\mathrm{ms}$ & & & \\
\hline Contender & 67 & 55 & 122 & 1,18 & 0,20 \\
\hline $\mathrm{EO} 2$ & 58 & 52 & 110 & 0,33 & 0,50 \\
\hline Emerit & 59 & 57 & 116 & 0,03 & 0,75 \\
\hline Red Kloud & 8 & 13 & 21 & 1,56 & 0,20 \\
\hline TG & 26 & 25 & 51 & 0,02 & 0,75 \\
\hline 422 & 140 & 141 & 281 & 0,00 & 0,90 \\
\hline 423 & 105 & 101 & 206 & 0,08 & 0,75 \\
\hline Borlotto Gigante & 17 & 18 & 35 & 0,03 & 0,75 \\
\hline Rousso & 7 & 4 & 11 & 0,82 & 0,30 \\
\hline $\mathrm{N}^{\circ} 15$ & 7 & 5 & 12 & 0,33 & 0,50 \\
\hline Totaux & 494 & 471 & 965 & 0,55 & 0,30 \\
\hline
\end{tabular}

$\mathrm{mf}=$ mâle fertile,

$\mathrm{ms}=$ mâle fertile

5 litres remplis d'un mélange de tourbe et de compost d'écorce de pin. Elles sont automatiquement irriguées plusieurs fois par jour avec une solution nutritive. La serre est protégée de l'intrusion des insectes pollinisateurs (bourdons et abeilles) par des mousselines de tissu synthétique.

\section{Repérage et observation du caractère de stérilité mâle}

Chaque plante est classée en fertile ou stérile par observation à la loupe de 4 ou 5 fleurs. Les plantes fertiles ont des stigmates couverts de pollen, les plantes stériles ont des stigmates totalement vierges et des anthères aplaties, non déhiscentes, ne contenant que du pollen avorté.

Pour déterminer le stade de l'avortement du pollen, des observations cytologiques plus fines ont été effectuées à partir d'anthères prélevées sur des boutons d'âge croissant depuis la préméioose jusqu'à l'anthère. Les anthères sont fixées dans une solution d'alcool acétique pendant 24 heures. Au-delà de ce temps, elles sont conservées dans l'éthanol à $70^{\circ}$. Pour l'observation, les anthères sont déposées dans une goutte de carmin acétique, écrasées délicatement entre lame et lamelle et observées au microscope optique. Les grains de pollen vivants ont un cytoplasme coloré en rose, les grains vides sont incolores.

\section{RÉSULTATS ET DISCUSSION}

\section{A. Déterminisme génétique}

Au tableau 1 figurent les résultats préliminaires et au tableau 2 les résultats de l'étude génétique complète, regroupés par parent mâle. Pour chacun d'eux les ségrégations mâle stériles/mâle fertiles obtenues en F1 sont statistiquement compatibles avec une répartition $1: 1$.

Toutes les descendances F2 issues d'autofécondation naturelle des $\mathrm{F} 1$ fertiles ont été trouvées uniformément fertiles.

De ces observations, nous pouvons tirer les deux conclusions suivantes : 
1) Les plantes F1 fertiles ne transmettent pas le caractère mâle stérile en autofécondation : elles sont donc homozygotes « normales ».

2) Les plantes $F 1$ stériles croisées avec n'importe quel génotype homozygote transmettent le caractère mâle stérile à la moitié de la descendance. Elles sont donc hétérozygotes pour le caractère mâle stérile et celui-ci est dominant.

Ces résultats sont en accord avec les cas de stérilités géniques dominantes déjà rapportés pour d'autres espèces. Ils peuvent être expliqués par l'action d'un gène dominant Ms commandant la stérilité. Les plantes mâle stériles seraient hétérozygotes Ms ms et les plantes fertiles homozygotes ms ms.

En croisement, les plantes mâle stériles donnent des gousses parfaitement remplies, le gène n'a donc pas d'incidence sur la mégasporogenèse et la fertilité femelle (fig. 1).

Il semble pourtant que dans les couples isogéniques en cours de constitution, les plantes mâle stériles soient légèrement moins vigoureuses et un peu plus tardives que les plantes fertiles.

\section{B. Etude cytologique}

Les observations cytologiques montrent qu'après digestion de l'enveloppe callosique qui entoure les microspores issues de la méiose on ne trouve chez les plantes stériles que quelques grains vides, alors que les plantes fertiles montrent des dizaines de microspores déjà colorées à ce stade où la taille du bouton floral est de $3 \mathrm{~mm}$. On peut donc conclure que la dégénérescence se produit à l'intérieur même de l'enveloppe callosique, donc immédiatement après la méiose, ce qui explique l'absence de turgescence des anthères, leur indéhiscence et l'absence totale de pollen sur le stigmate.

\section{Exception}

Il faut néanmoins signaler l'exception que constitue la variété Copau (origine bulgare). Les rétrocroisements poursuivis avec cette variété font apparaître, à partir du $2^{\mathrm{e}}$ rétrocroisement, des plantes "stériles " possédant un peu de pollen colorable, qui peuvent de ce fait donner quelques graines en autofécondation et en croisement lorsqu'elles sont utilisées comme mâles. Ces plantes semi-stériles sont bien hétérozygotes pour le gène Ms, car lorsqu'elles sont utilisées comme femelles en croisement avec une autre variété, elles transmettent le caractère mâle stérile à 50 p. 100 , comme le feraient des plantes totalement mâle stériles. Par contre, lorsque ces plantes semi-stériles ont été utilisées comme mâles, en croisement avec des plantes mâle stériles provenant d'un autre système génétique où la stérilité mâle est commandée par un gène récessif, aucune plante stérile ou semi-stérile n'a été observée sur une dizaine de plantes F1 : il n'est donc pas certain que le gène puisse être transmis par le pollen et que l'on puisse le transférer sur un autre cytoplasme.

\section{Utilisation en sélection}

L'utilisation des stérilités mâle géniques en sélection a été discutée par plusieurs auteurs (GILMORE, 1964 ; BRIM \& STUBERS, 1973 ; RAMAGE, 1977 ; SORRELlS \& FrITZ, 1982). Nous pensons que les deux avantages d'une stérilité génique dominante sur une stérilité génique récessive se situent respectivement au début et à la fin de la phase d'intercroisements (croisements pyramidaux ou circulaires) d'un programme de sélection récurrente.

1) Les plantes mâle stériles apparaissent dès la $F 1$ des premiers croisements. Ceci permet de disposer à chaque génération d'une forte proportion de plantes stériles, alors que dans le cas d'une stérilité récessive, il faut une génération d'autofécondation supplémentaire pour trouver des plantes stériles. Par la suite, les deux systèmes deviennent progressivement équivalents, car les croisements des plantes mâle stériles (homozygotes dans le système de stérilité récessive, mais hétérozygotes dans le système de stérilité dominante) par des plantes fertiles (hétérozygotes dans le système récessif, mais homozygotes dans le système dominant) donnent 50 p. 100 de plantes mâle stériles.

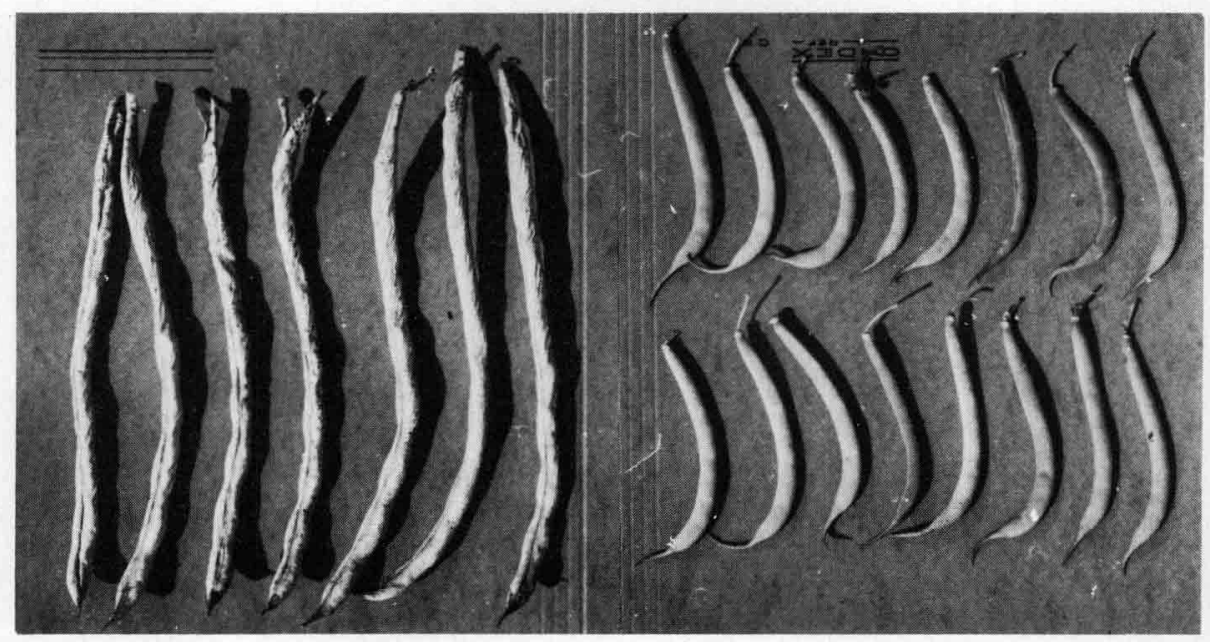

Figure 1

Gousses non fécondées parthénocarpiques (droite) et gousses fécondées par croisement (gauche) d'un même génotype mâle stérile.
Parthenocarpic unfertilized pods (right) and cross-fertilized pods (left) on the same male-sterile genotype. 
2) Après les cycles d'intercroisements, le système dominant livre directement des descendances uniformément fertiles, ce qui permet une évaluation précoce des caractères quantitatifs. Par contre, les mêmes descendances dans un système de stérilité récessive seront en ségrégation pour la stérilité mâle, ce qui risque de fausser l'évaluation précoce. Il sera donc nécessaire d'éliminer le caractère de stérilité mâle avant toute évaluation, ce qui entraîne des retards, donc une perte d'efficacité.

Il faut toutefois signaler, pour le système dominant décrit ici, un inconvénient éventuellement lourd de conséquences. Jusqu'à présent, il n'a pas été possible de dissocier le gène du cytoplasme où il est apparu. Ceci implique que l'ensemble des descendances possèderont le même cytoplasme, celui de la variété Valja. On connaît les dangers éventuels de l'uniformité cytoplasmique.

Nous proposons, compte tenu des systèmes précé- demment décrits, de donner à ce gène le nom et la définition qui suivent :

\section{Nom : Ms8}

Définition: gène dominant de stérilité mâle s'exprimant au stade tétrade de la microsporogenèse et entrâ̂nant une dégénérescence totale des quatre microspores, sans effet apparent sur la fertilité femelle.

\section{REMERCIEMENTS}

Les auteurs remercient Mademoiselle Risser et Monsieur HeRvÉ de leurs critiques constructives.

Reçu le 9 octobre 1986 Accepté le 11 avril 1987

\section{REFERENCES BIBLIOGRAPHIQUES}

Agbo, F. M. O., Wood D. R., 1977. Observation of male-sterility in dry beans. Annu. Rept. Bean Imp. Coop., $20: 49-50$.

Allison D. C., Fisher W. D., 1964. A dominant gene for malesterility in upland cotton. Crop Sci., $4: 548-549$.

Bannerot H., 1980. Five cases of male-sterility in beans. Annu. Rept. Bean Imp. Coop., vol. 23, p. 121 A.

Bassett M. J., Hung L., 1982. Induction of semi-sterility mutations in common bean, Phaseolus vulgaris L. J. Amer. Soc. Hort. Sci., 107 (5) : 871-874.

Bell J. M., 1986. Etude génétique, cytologique et physiologique de différentes stérilités mâle géniques et cytoplasmique chez Phaseolus vulgaris $L$. Thèse Univer. Paris $11, \mathrm{n}^{\circ}$ ordre 111,177 p., 1986.

Brim C. A., Stubers C. W., 1973. Application of genetic malesterility to recurrent schemes in soybeans. Crop Sci., 13 : 528-530.

Bowman D. T., Weaver J. B. Jr., 1979. Analysis of a dominant male-sterile character in upland cotton. II. Genetic Studies. Crop Sci., $19: 628-630$.

Gilmore E. C. Jr., 1964. Suggested method of using reciprocal recurrent selection in some naturally self-pollinated species. Crop Sci., $4: 323-325$.

Ibrahim A. M., Coyne D. P., Emory G., 1974. Male-sterility and stigma shape alteration in Phaseolus vulgaris. Annu. Rept. Bean Imp. Coop., $17: 48-49$.

Mutschler M. A., Bliss F. A., 1980. Genic male-sterility in the common bean (Phaseolus vulgaris L.). J. Amer. Soc. Hort. Sci., 105 (2) : 202-205.
Ramage R. T., 1977. Varietal improvement of wheat through malesterile facilitated recurrent selection. Technical Bulletin, ASPAC Food and Fertilizer Technology Center, $\mathrm{n}^{\circ} 37$.

Ryder E. J., 1963. An epistatically controlled pollen sterile in lettuce (Lactuca sativa L.). Proc. Amer. Soc. Hort. Sci., 83 : 585-589.

Sasakuma T., Maan S. S., Williams N. I., 1978. EMS-induced male-sterile mutants in euplasmic and alloplasmic common wheat. Crop Sci., $18: 850-853$.

Singh S. P., White J. W., Guttierrez J. A., 1980. Male-sterile in dry beans. Annu. Rept. Bean Imp. Coop., 23 : 55-57.

Sorrells M. E., Fritz S. E., 1982. Application of a dominant malesterile allele to the improvement of self-pollinated crops. Crop Sci. 22 : 1033-1035.

Turcotte E. L., Feaster C. V., 1985. Inheritance of malesterile mutant MS 12 in American Pima cotton. Crop Sci., 25 : 688690.

Van Rheenen M. A., Muigai S. G. S., Kitivo D. K., 1979. Malesterility in beans (Phaseolus vulgaris L.) Euphytica, 28 : 761-763.

Weaver J. B. Jr., Ashlay T., 1971. Analysis of a dominant gene for male-sterility in upland cotton, Gossypium hirsutum L. Crop Sci., $11: 596-598$.

Wyatt J. E., 1984. An indehiscent anther mutant in the common bean, Phaseolus vulgaris L. J. Amer. Soc. Hort. Sci., 109 (4) : 481-487. 